\title{
Pathological factors related to lymph node metastasis of submucosally invasive gastric cancer: criteria for additional gastrectomy after endoscopic resection
}

\author{
Mototsugu Fujii · Yutaro Egashira $\cdot$ Hiroshi Akutagawa • \\ Tsukasa Nishida · Toshikatsu Nitta • Go Edagawa • \\ Yoshitaka Kurisu • Yuro Shibayama
}

Received: 1 July 2012/Accepted: 4 November 2012/Published online: 23 November 2012

(C) The International Gastric Cancer Association and The Japanese Gastric Cancer Association 2012

\begin{abstract}
Background There are currently no universally accepted indications and criteria for additional gastrectomy after endoscopic resection of submucosally invasive cancer. The purpose of the present study was to establish accurate indications and criteria for such additional gastrectomy on the basis of lymph node metastasis risk.

Methods We investigated 130 submucosally invasive gastric cancers and analyzed the pathological risk factors for lymph node metastasis. The tumors were evaluated for pathological factors in the area of invasion, and factors were compared between the cases with lymph node metastasis and those without.

Results Univariate logistic regression analysis showed that tumor minor axis length, depth of invasion, histological classification of the area of submucosal invasion, absence of lymphoid infiltration, ulceration or scar in the lesion, and lymphatic and venous invasion are statistically significant risk factors for lymph node metastasis. Multivariate logistic regression analysis showed that the absence of lymphoid infiltration and the presence of lymphatic invasion are statistically significant as risk factors for lymph node metastasis.

Conclusions We present a scoring system on the basis of the pathological criteria tested in this study. Our findings enable more accurate identification of patients who should undergo additional gastrectomy after endoscopic resection.
\end{abstract}

M. Fujii $(\bowtie) \cdot$ Y. Egashira · H. Akutagawa - T. Nishida ·

G. Edagawa · Y. Kurisu · Y. Shibayama

Department of Pathology, Osaka Medical College,

Daigaku-machi, Takatsuki, Osaka 569-8686, Japan

e-mail: fujii-mo@mc.pref.osaka.jp

T. Nitta

Shiroyama Hospital, Habikino, Habikino,

Osaka 583-0872, Japan
Keywords Endoscopic submucosal dissection .

Submucosally invasive gastric cancers - Additional gastrectomy after endoscopic resection - Pathological risk factors for lymph node metastasis

\section{Background}

Endoscopic resection, including endoscopic submucosal dissection, has been established as a standard means of treatment of differentiated gastric mucosal cancer. Application of this technique has now extended to undifferentiated cancer and submucosally invasive gastric cancers [1]. In some cases, submucosally invasive gastric cancers are often endoscopically resected intentionally or unintentionally. Regional lymph node metastasis has been reported to occur in about $10 \%$ of patients with submucosally invasive gastric cancers [2-5]. Therefore, endoscopic resection alone is considered insufficient as radical treatment of such cancers, which may metastasize to lymph nodes. Hence, additional gastrectomy with lymph node dissection is required. Unfortunately, however, there is no consensus regarding indications for additional gastrectomy following endoscopic resection of submucosally invasive gastric cancer [4-6]. In many cases, submucosally invasive gastric cancers persisting after endoscopic procedures require additional surgery. As a consequence, many patients have cancer-free lymph nodes resected, i.e., they are overtreated, whereas some patients who undergo no additional gastrectomy exhibit recurrent lymph node metastasis.

Conventionally, many factors including tumor axis length, submucosal invasion (distance, width, and area), lymphatic invasion, venous invasion, histological classification, mucus phenotype, ulceration or scar in the cancer 
lesion, and cancer invasion style and area have been considered to be pathological risk factors for lymph node metastasis of gastric cancer [2-6]. However, none of these risk factors provides $100 \%$ sensitivity for detecting metastasis, and each risk factor has low individual specificity for metastasis. Several risk factors must therefore be combined to obtain useful criteria for performing of additional gastrectomy of submucosally invasive gastric cancers on the basis of prediction of lymph node metastasis.

In our study, pathological factors involved in lymph node metastasis of submucosally invasive gastric cancers were examined with the goal of establishing criteria for performing additional gastrectomy on the basis of prediction of lymph node metastasis following endoscopic resection.

\section{Methods}

The present study included 130 patients with submucosally invasive gastric cancers who underwent gastrectomy with D1 or higher lymph node dissection between 1998 and 2010 at Osaka Medical College, Japan, for whom satisfactory clinicopathological data were available. Patients who underwent additional gastrectomy following endoscopic resection of submucosally invasive gastric cancers were excluded from the present study, because many such patients with D1 lymph node dissection are insufficiently treated, making it difficult to accurately evaluate lymph node metastasis.

Surgically excised gastric tissues containing submucosally invasive cancer were fixed in $20 \%$ buffered formalin, cut into 5-mm-wide sections in the direction of the gastric longitudinal axis, and embedded in paraffin. The following pathological factors were examined, using hematoxylin and eosin (H\&E)-stained specimens providing the largest cut surface of gastric cancer: length of tumor axes (major and minor), tumor area, depth and width of submucosal invasion, area of submucosal invasion, histological classification, mucus phenotype, ulceration or scar in the lesion, cancer invasion style, lymphocytic infiltration, lymphatic invasion, and venous invasion. Definitions, classifications, and measurement methods for each of these factors are described below.

Length of tumor axes (major and minor) and tumor area

The largest macroscopic dimension of each cancer lesion was defined as the major axis, whereas the largest dimension orthogonally crossing the major axis was defined as the minor axis. The tumor area was defined as pai $/ 4 \times$ major axis $\times$ minor axis.
Depth and width of submucosal invasion

The depth and width of submucosal invasion were measured according to the method for measurement of the depth of submucosal invasion of colonic cancer proposed in the General Rules for Clinical and Pathological Studies on Cancer of the Colon, Rectum, and Anus [7].

Area of submucosal invasion in cancer

The product of the depth and width of submucosal invasion in cancer was defined as the area of submucosal cancer invasion.

\section{Histological classification}

Cancer tissue (intramucosally and at the site of submucosal invasion) limited to tubular and papillary adenocarcinoma was classified as differentiated type, whereas that containing any features of poorly differentiated adenocarcinoma, signet-ring cell carcinoma, or mucinous adenocarcinoma was classified as undifferentiated type.

Mucus phenotype

Cancer tissue was immunostained with human gastric mucin (HGM, crypt epithelium type) and MUC 6 (pyloric gland type) as gastric mucus phenotype markers and MUC 2 (goblet cell type) and CD10 (small intestine type) as intestinal mucus phenotype markers. When more than $5 \%$ of tumor cells were positive for either HGM or MUC 6, the tumor was classified as a gastric-type cancer. When more than $5 \%$ of tumor cells were positive for either MUC 2 or CD10 and negative for both HGM and MUC 6, the tumor was classified as an intestinal-type cancer.

\section{Ulceration or scar in the cancer lesion}

The presence of open ulcer or ulcer scars on tumors was determined.

\section{Cancer invasion style}

Invasion style of submucosally invasive cancer at the site of invasion was classified as infiltrative growth pattern (INF) a, b, or c according to the Japanese Classification of Gastric Carcinoma [6].

Lymphocytic infiltration

Cases in which a clear lymphoid follicle formation was observed at the site of submucosal invasion (lymphocytes were more common than tumor cells) were labeled positive 
for lymphocytic infiltration. All other cases were labeled negative for lymphocytic infiltration.

Lymphatic invasion

Lymphatic invasion was confirmed by H\&E staining. Falsepositive cases were reconfirmed by D2-40 immunostaining.

\section{Venous invasion}

In all cases, venous invasion was determined by elastica van Gieson staining.

\section{Statistical analysis}

Relationships between the dependent variable of lymph node metastasis and the pathological factors as explanatory variables were examined by the logistic regression method, using Statview Version 5.0 software [8]. First, all pathological factors were subjected to univariate analysis to calculate $P$ values and odds ratios. Then, those factors with $P<0.1$ on univariate analysis were subjected to multivariate analysis to calculate $P$ values and odds ratios. Findings with $P<0.1$ were considered significant.

\section{Results}

The ratio of male to female patients was $44: 86$. The mean age of patients in this study was $65.1 \pm 30.1$ years. By gross morphology, the cancers included 9 cases of protruding type (type IIa, $n=4$; type I, $n=4$; type IIa + I, $n=1$ ). In addition, 91 cases were of excavated type (type IIc, $n=76$; type IIc + III, $n=14$; type IIc + IIb, $n=1$ ). Thirty cases were of combined protruding and excavated type (IIa + IIc, $n=26$; type IIc + IIa, $n=4)$.

Factor analysis

1. Of 130 patients with submucosally invasive cancer, 22 $(16.9 \%)$ were positive for lymph node metastasis [n(+)], whereas $108(83.1 \%)$ were negative for lymph node metastasis $[n(-)]$ (Table 1$)$.

2. The mean major axis length of tumors in all patients was $3.7 \mathrm{~cm}$. The mean major axis length of tumors was $4.4 \mathrm{~cm}$ in $n(+)$ cases and $3.5 \mathrm{~cm}$ in $n(-)$ cases (Table 1). The mean minor axis length in all patients was $2.4 \mathrm{~cm}$. The mean minor axis length was $3.2 \mathrm{~cm}$ in $n(+)$ cases and $2.3 \mathrm{~cm}$ in $n(-)$ cases. The mean minor axis length of tumors in $n(+)$ cases was significantly greater than that in $n(-)$ cases $(P<0.05)$ (Table 1). The minimum minor axis length in $n(+)$ cases was $0.8 \mathrm{~cm}$. On logistic regression analysis of
Table 1 Relationship between tumor axes (major and minor), area, and lymph node metastasis

\begin{tabular}{llll}
\hline & $n(+)$ & $n(-)$ & Total \\
\hline Cases & 22 & 108 & 130 \\
& $(16.9 \%)$ & $(83.1 \%)$ & $(100 \%)$ \\
Mean major axis $(\mathrm{cm}) *$ & 4.4 & 3.5 & 3.7 \\
Mean minor axis $(\mathrm{cm}) * *$ & 3.2 & 2.3 & 2.4 \\
Mean area $\left(\mathrm{cm}^{2}\right)^{*}$ & 17.4 & 8.7 & 10.5 \\
Minor axis $\geq 2 \mathrm{~cm}^{*}$ & 15 & $50(76.9 \%)$ & $65(100 \%)$ \\
Minor axis $<2 \mathrm{~cm}$ & $(23.1 \%)$ & & \\
$* P<0.05$ & $7(10.8 \%)$ & $58(89.2 \%)$ & $65(100 \%)$ \\
$* * P<0.1$ & & &
\end{tabular}

Table 2 Relationship between depth, width, area of submucosal invasion, and lymph node metastasis

\begin{tabular}{llll}
\hline & $n(+)$ & $n(-)$ & Total \\
\hline Cases & $22(16.9 \%)$ & $108(83.1 \%)$ & $130(100 \%)$ \\
Mean depth $(\mu \mathrm{m})^{*}$ & 2,444 & 1,473 & 1,637 \\
Mean width $(\mu \mathrm{m})^{*}$ & 9,946 & 5,287 & 6,075 \\
Mean area $\left(\mathrm{mm}^{2}\right)^{*}$ & 33.9 & 12.3 & 16.0 \\
$\begin{array}{c}\text { Depth of submucosal } \\
\text { invasion } \geq 2,000 \mu \mathrm{m} *\end{array}$ & $13(31.7 \%)$ & $28(68.3 \%)$ & $41(100 \%)$ \\
$\begin{array}{l}\text { Depth of submucosal } \\
\text { invasion }<2,000 \mu \mathrm{m}\end{array}$ & $9(10.1 \%)$ & $80(89.9 \%)$ & $89(100 \%)$ \\
$* P<0.05$ & & & \\
\hline
\end{tabular}

the minor axis length, the patients were divided into two groups with longer and shorter minor axis. The odds ratio was highest when the cutoff value was set at $2.0 \mathrm{~cm}$. The prevalence of lymph node metastasis was significantly higher in patients with submucosally invasive cancer with a minor axis length $\geq 2.0 \mathrm{~cm}$ than in patients with submucosally invasive cancer with a minor axis length $<2.0 \mathrm{~cm}(P<0.05$; Table 1$)$.

Mean tumor area (pai $/ 4 \times$ major axis $\times$ minor axis) was $1,045 \mathrm{~mm}^{2}$ overall, $1,739 \mathrm{~mm}^{2}$ in the $n(+)$ cases, and $871 \mathrm{~mm}^{2}$ in the $n(-)$ cases. The area of submucosal invasion in cancer was significantly greater in $n(+)$ cases than in $n(-)$ cases $(P<0.1$; Table 1$)$.

3. Mean depth of submucosal cancer invasion was $1,637 \mu \mathrm{m}$ overall, $2,444 \mu \mathrm{m}$ in the $n(+)$ cases, and $1,473 \mu \mathrm{m}$ in the $n(-)$ cases. Mean depth of submucosal cancer invasion was significantly greater in $n(+)$ cases than in $n(-)$ cases $(P<0.05)$ (Table 2). On logistic regression analysis of depth of invasion, patients were divided into two groups: deep and shallow. The odds ratio was highest when the cutoff value was set at $2,000 \mu \mathrm{m}$. These findings suggested that use of $2,000 \mu \mathrm{m}$ for depth of 
submucosal invasion as a criterion for risk of lymph node metastasis was reasonable.

4. Mean width of submucosal cancer invasion was $6,075 \mu \mathrm{m}$ overall; it was $9,946 \mu \mathrm{m}$ in the $n(+)$ cases and $5,287 \mu \mathrm{m}$ in the $n(-)$ cases. Width of invasion was significantly greater in $n(+)$ cases than in $n(-)$ cases $(P<0.05$; Table 2$)$.

5. Mean area of submucosal cancer invasion was $16.0 \mathrm{~mm}^{2}$ overall, $33.9 \mathrm{~mm}^{2}$ in the $n(+)$ cases, and $12.3 \mathrm{~mm}^{2}$ in the $n(-)$ cases. Area of invasion was significantly greater in the $n(+)$ cases than in the $n(-)$ cases $(P<0.05$; Table 2$)$.

6. Intramucosal cancer tissue was of the differentiated type in 38 patients and undifferentiated type in 92 patients, with prevalence of lymph node metastasis in these two groups of 13.2 and $18.5 \%$, respectively. Submucosal cancer tissue at the site of invasion was of differentiated type in 38 patients and undifferentiated type in 92 patients; the prevalence of lymph node metastasis in these two groups was 5.3 and $21.7 \%$, respectively. The prevalence of lymph node metastasis was significantly higher in patients with undifferentiated submucosal cancer at the site of invasion than in patients with well-differentiated submucosal cancer at the site of invasion $(P<0.05$; Table 3$)$.

7. According to mucus phenotype, intestinal-type cancers were observed in 45 patients and gastric-type cancers were observed in 85 patients; the prevalence of lymph node metastasis in these groups was $11.1 \%$ and $20.0 \%$, respectively.

8. There were 80 patients with submucosal invasive cancer complicated by ulceration (scar), with a rate of lymph node metastasis of $22.5 \%$. In contrast, there were 50 patients with submucosal invasive cancer not complicated by ulceration (scar), with a rate of lymph node metastasis of $8.0 \%$. The prevalence of lymph node metastasis was significantly higher in patients with submucosal invasive cancer complicated by ulceration (scar) than in those with cancer not so complicated $(P<0.05$; Table 3$)$.

9. The mode of invasion of submucosal invasive cancer was INF a or b in 99 patients, with a rate of lymph node metastasis of $14.1 \%$. INF c mode was observed in 31 patients, with a rate of lymph node metastasis of $25.8 \%$ (Table 3).

10. Lymphocytic infiltration was observed in 50 patients, with a rate of lymph node metastasis of $2.0 \%$. In contrast, lymphocytic infiltration was absent in 80 patients, whose rate of lymph node metastasis was $26.2 \%$. The prevalence of lymph node metastasis was significantly lower in patients with lymphocytic infiltration $(+)$ than in those without infiltration $(P<0.05$; Table 3$)$.
Table 3 Relationship between pathological factors and lymph node metastasis of submucosal invasive gastric cancer

\begin{tabular}{|c|c|c|c|}
\hline Pathological factors & $n(+)$ & $n(-)$ & Total \\
\hline \multicolumn{4}{|c|}{$\begin{array}{l}\text { Histological classification } \\
\text { (intramucosal) }\end{array}$} \\
\hline Differentiated & $5(13.2 \%)$ & $33(86.8 \%)$ & 38 \\
\hline Undifferentiated & $17(18.5 \%)$ & $75(81.5 \%)$ & 92 \\
\hline \multicolumn{4}{|c|}{$\begin{array}{l}\text { Histological classification } \\
\text { (submucosal)* }\end{array}$} \\
\hline Differentiated & $2(5.3 \%)$ & $36(94.7 \%)$ & 38 \\
\hline Undifferentiated & $20(21.7 \%)$ & $72(78.3 \%)$ & 92 \\
\hline \multicolumn{4}{|l|}{ Mucus phenotype } \\
\hline Intestinal type & $5(11.1 \%)$ & $40(88.9 \%)$ & 45 \\
\hline Gastric type & $17(20.0 \%)$ & $68(80.0 \%)$ & 85 \\
\hline \multicolumn{4}{|c|}{ Ulcer or scar in cancer lesion* } \\
\hline$(+)$ & $18(22.5 \%)$ & $62(77.5 \%)$ & 80 \\
\hline$(-)$ & $4(8.0 \%)$ & $46(92.0 \%)$ & 50 \\
\hline \multicolumn{4}{|c|}{ Style of invasion of cancer } \\
\hline$a, b$ & $14(14.1 \%)$ & $85(85.9 \%)$ & 99 \\
\hline $\mathrm{c}$ & $8(25.8 \%)$ & $23(74.2 \%)$ & 31 \\
\hline \multicolumn{4}{|c|}{ Lymphocytic infiltration* } \\
\hline$(+)$ & $1(2.0 \%)$ & $49(98.0 \%)$ & 50 \\
\hline$(-)$ & $21(26.2 \%)$ & $59(73.8 \%)$ & 80 \\
\hline \multicolumn{4}{|l|}{ Lymphatic invasion* } \\
\hline$(+)$ & $19(32.2 \%)$ & $40(67.8 \%)$ & 59 \\
\hline$(-)$ & $3(4.2 \%)$ & $68(95.8 \%)$ & 71 \\
\hline \multicolumn{4}{|l|}{ Venous invasion* } \\
\hline$(+)$ & $7(30.4 \%)$ & $16(69.6 \%)$ & 23 \\
\hline$(-)$ & $15(14.0 \%)$ & $92(86.0 \%)$ & 107 \\
\hline
\end{tabular}

* $P<0.05$

11. There were 59 lymphatic invasion-positive patients, with the rate of lymph node metastasis being $32.2 \%$. Among the 71 lymphatic invasion-negative patients, the rate of lymph node metastasis was $4.2 \%$. This rate was significantly higher in lymphatic invasionpositive than in lymphatic invasion-negative patients $(P<0.05$; Table 3$)$.

12. There were 23 venous invasion-positive patients, with a rate of lymph node metastasis of $30.4 \%$. Among the 107 venous invasion-negative patients, the rate of lymph node metastasis was $14.0 \%$. The rate of lymph node metastasis was significantly higher in venous invasion-positive than in venous invasionnegative patients $(P<0.05$; Table 3$)$.

Statistical analysis

Of the foregoing pathological factors examined by univariate analysis, the following factors exhibited a significant correlation with lymph node metastasis: tumor minor 
Table 4 Relationship between pathological factors and lymph node metastasis of submucosal invasive gastric cancer (statistical analysis)

\begin{tabular}{|c|c|c|c|c|}
\hline \multirow[t]{2}{*}{ Pathological factors } & \multicolumn{2}{|c|}{$\begin{array}{l}\text { Univariate } \\
\text { analysis }\end{array}$} & \multicolumn{2}{|c|}{$\begin{array}{l}\text { Multivariate } \\
\text { analysis }\end{array}$} \\
\hline & $P$ value & $\begin{array}{l}\text { Odds } \\
\text { ratio }\end{array}$ & $P$ value & $\begin{array}{l}\text { Odds } \\
\text { ratio }\end{array}$ \\
\hline Minor axis of tumor & 0.067 & 2.49 & $P \geq 0.1$ & - \\
\hline $\begin{array}{l}\text { Depth of submucosal } \\
\text { invasion }\end{array}$ & 0.008 & 3.59 & $P \geq 0.1$ & - \\
\hline $\begin{array}{l}\text { Histological classification } \\
\text { (intramucosal) }\end{array}$ & $P \geq 0.1$ & - & - & - \\
\hline $\begin{array}{l}\text { Histological classification } \\
\text { (submucosal) }\end{array}$ & 0.036 & 5.0 & $P \geq 0.1$ & - \\
\hline Mucus phenotype & $P \geq 0.1$ & - & - & - \\
\hline $\begin{array}{l}\text { Ulcer or scar (in cancer } \\
\text { lesion) }\end{array}$ & 0.039 & 3.33 & $P \geq 0.1$ & - \\
\hline Style invasion of cancer & $P \geq 0.1$ & - & - & - \\
\hline Lymphocytic infiltration & 0.01 & 5.26 & 0.005 & 7.94 \\
\hline Lymphatic invasion (1 y) & 0.0003 & 10.76 & 0.0035 & 8.07 \\
\hline Venous invasion (v) & 0.063 & 2.68 & $P \geq 0.1$ & - \\
\hline
\end{tabular}

axis length, tumor area, submucosal invasion (depth, width, and area), histological classification of submucosal cancer (undifferentiated) at site of invasion, lymphocytic infiltration, ulceration or scar in the lesion, lymphatic invasion, and venous invasion. Of these factors, lymphocytic infiltration alone exhibited a negative correlation. The risk of lymph node metastasis was significantly lower in patients with lymphocytic infiltration than in those without it (Table 4). Those pathological factors significant on univariate analysis were then subjected to multivariate analysis. Results showed that significant, independent risk factors for lymph node metastasis included lymphatic invasion and lymphocytic infiltration (Table 4).

\section{Scoring to predict lymph node metastasis}

Pathological factors extracted from the univariate analysis were scored to predict the onset of lymph node metastasis on the basis of the total score. Of the two pathological factors extracted by univariate analysis, tumor minor axis length and tumor area, each representing the size of the tumor, minor axis length was selected for predictive scoring because of its high odds ratio and simplicity of measurement. Similarly, submucosal invasion depth was selected as a scoring factor among the three pathological factors of depth, width, and area of invasion. Prediction of lymph node metastasis was thus scored using seven pathological factors: tumor minor axis length, depth of submucosal invasion, histological classification (undifferentiated) of submucosal cancer at the site of invasion, lymphocytic infiltration, ulceration or scar in the
Table 5 Scoring to predict lymph node metastasis

\begin{tabular}{lll}
\hline Score -2 & Score +1 & Score +2 \\
\hline $\begin{array}{c}\text { Lymphocytic } \\
\text { infiltration }(+)\end{array}$ & $\begin{array}{c}\text { Histological classification } \\
\text { undifferentiated (submucosal) }\end{array}$ & $\begin{array}{c}\text { Lymphatic } \\
\text { invasion } \\
(+)\end{array}$ \\
& Minor axis $\geq 2 \mathrm{~cm}$ & \\
& Depth of submucosal invasion \\
& $\geq 2,000 \mu \mathrm{m}$ \\
& Ulcer or scar (in cancer lesion) \\
& Venous invasion $(+)$ & \\
\hline
\end{tabular}

lesion, lymphatic invasion, and venous invasion. Results of studies in the literature were taken into consideration in scoring as follows $[9,10]$. Two pathological factors, lymphocytic infiltration and lymphatic invasion, were found to be independent on multivariate analysis. These factors were scored as follows: +2 for lymphatic invasion and -2 for lymphocytic infiltration positivity, which was considered a lymph node metastasis-inhibiting factor. Five pathological factors [minor axis length $\geq 2 \mathrm{~cm}$, submucosal invasion depth $\geq 2,000 \mu \mathrm{m}$, histological classification (undifferentiated) of submucosal cancer at the site of invasion, ulceration or scar in the lesion, and venous invasion], which were found not to be independent on multivariate analysis, were scored +1 each when present (Table 5).

The total score of pathological factors ranged from -2 to +7 in patients included in the present study. To determine the minimum total score at which lymph node metastasis may occur, and to aid in deciding whether additional gastrectomy should be performed, sensitivity and specificity of the prediction of lymph node metastasis were calculated at each score within this range. Sensitivity was found to be $100 \%$. Specificity reached maximum (63.9\%) when a total score of $\geq 3$ was regarded as indicating risk of lymph node metastasis requiring additional gastrectomy (Table 6).

Figure 1 shows scoring to predict lymph node metastasis on the basis of the results of this analysis.

\section{Discussion}

Many empirical studies have shown that longer axes of submucosally invasive gastric cancer tumors tend to be significantly associated with an increased prevalence of lymph node metastasis. In the present study, instead of the larger dimension (major axis), we focused on the minor axis, which correlated significantly with lymph node metastasis. This finding suggests that the prevalence of lymph node metastasis may be low for elongated submucosally invasive cancers with larger differences between 
Table 6 Determine the minimal total score at which lymph node metastasis may occur and decide whether additional gastrectomy should be performed

\begin{tabular}{ll} 
Observation & $\begin{array}{l}\text { Additional } \\
\text { gastrectomy }\end{array}$ \\
\hline
\end{tabular}

Sensitivity $100 \%$, specificity $41.7 \%$

$\begin{array}{lrl}n(-) & 45 & 63 \\ n(+) & 0 & 22\end{array}$

Total score $\geq 2$

Sensitivity $100 \%$, specificity $63.9 \%$

$\begin{array}{lrl}n(-) & 69 & 39 \\ n(+) & 0 & 22\end{array}$

Total score $\geq 3$

Sensitivity $76.9 \%$, specificity $81.5 \%$

$n(-)$

$n(+)$

5

17

Total score $\geq 4$ lengths of major and minor axes, even when the greatest dimension (major axis) is large. Circular tumor lesions with longer axes thus appear to present a higher risk of lymph node metastasis.

Depth of submucosal cancer invasion has previously been found to be an important risk factor for lymph node metastasis [1-6]. In the present study in which depth of invasion was divided into two groups (i.e., greater and smaller) for the purposes of logistic regression analysis, the odds ratio was highest when the depth of invasion cutoff point was set at $2,000 \mu \mathrm{m}$. This finding suggested that $2,000 \mu \mathrm{m}$ can be used as a criterion to predict risk of lymph node metastasis. However, the single factor of depth of submucosal invasion of cancer, which is less sensitive in predicting lymph node metastasis, should be combined with other risk factors to improve prediction.

When gastric cancer is classified histologically as poorly differentiated adenocarcinoma or a mixture of well- and

Fig. 1 Scoring system for additional gastrectomy following endoscopic resection based on prediction of lymph node metastasis

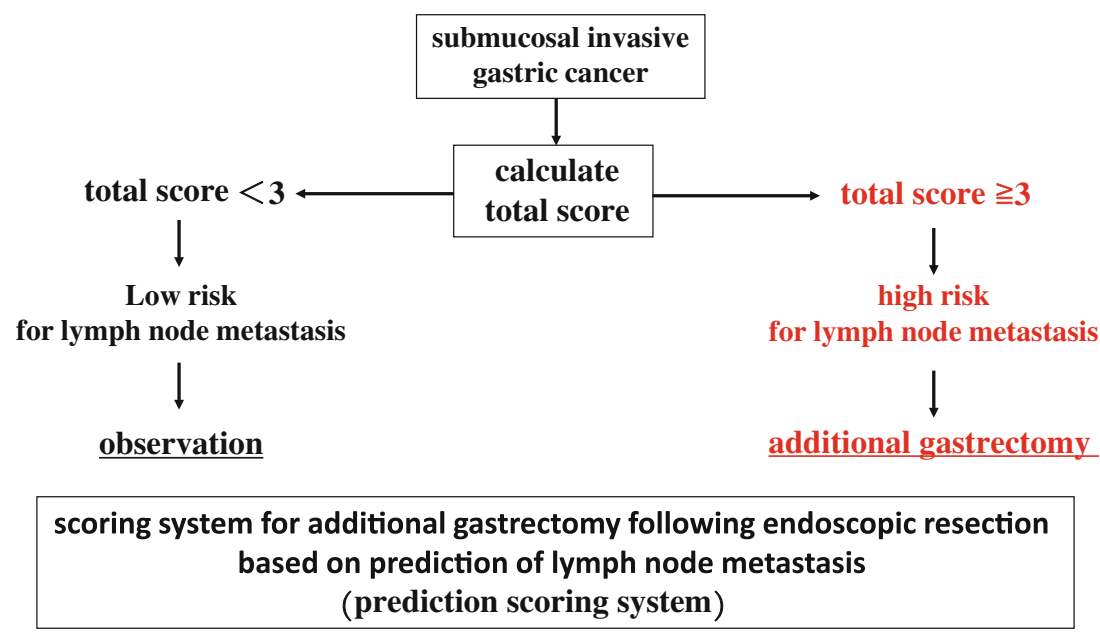

Table 7 Comparison pathological factor-based scoring system and the other criteria for additional gastrectomy

Observation

Pathological factor-based scoring system

$n(-)$

$n(+)$

Not $<500 \mu \mathrm{m}$ depth of invasion

$n(-)$

35

$n(+)$

3

Additional

gastrectomy

\begin{tabular}{lccc}
\hline Criteria & Sensitivity (\%) & Specificity (\%) & Diagnostic accuracy $(\%)$ \\
\hline Pathological factor based scoring system & 100 & 63.9 & 70.0 \\
Not $<500 \mu \mathrm{m}$ depth of invasion & 86.4 & 32.4 & 41.5 \\
Not $<1,000 \mu \mathrm{m}$ depth of invasion & 68.2 & 49.1 & 52.3 \\
Not $<500 \mu \mathrm{m}$ depth of invasion and lymphatic invasion & 100 & 30.6 & 42.3 \\
\hline
\end{tabular}




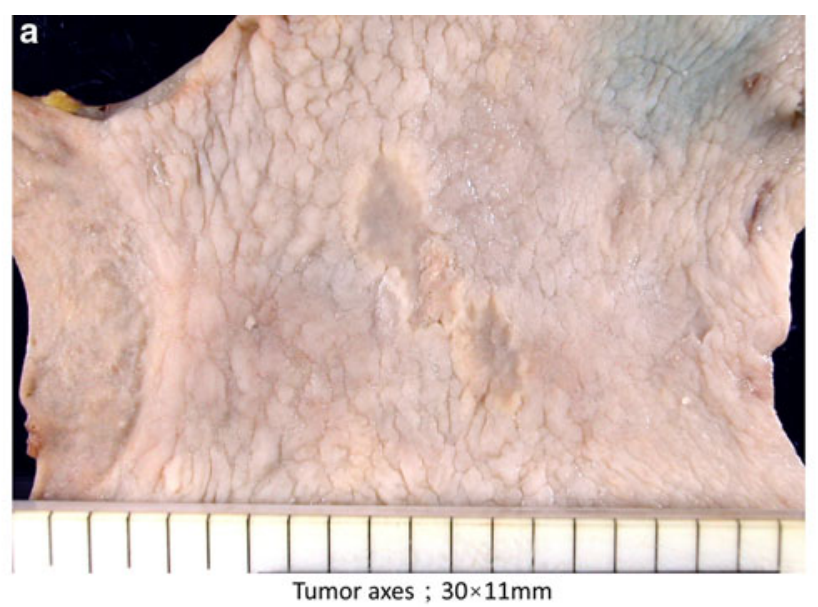

b

depth of submucosal cancer invasion $300 \mu \mathrm{m}$

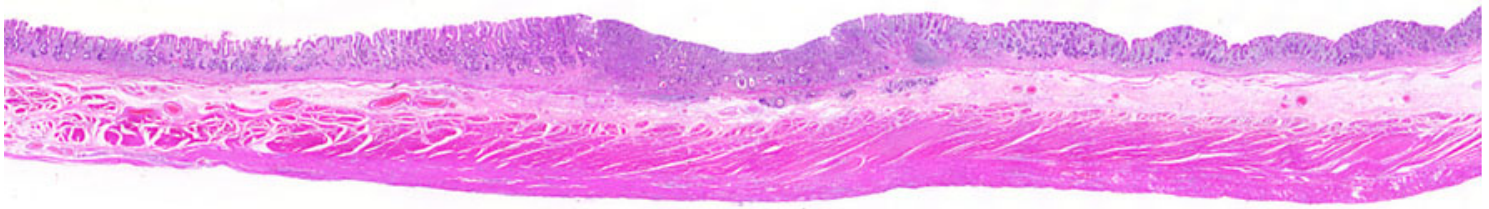

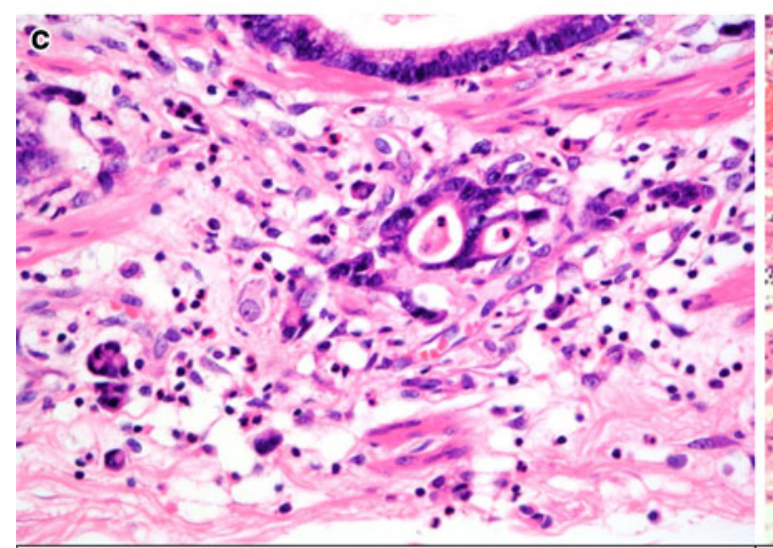

submucosal cancer tissue; undifferentiated type

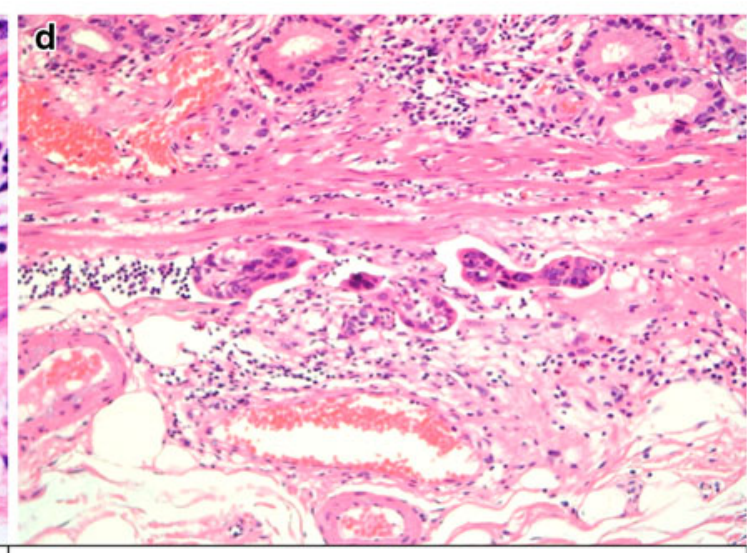

Lymphatic invasion positive

Fig. 2 Case 1, which was positive for lymph node metastasis (total score: +3 ). a Tumor size (score: \pm 0 ). b Depth of submucosal cancer invasion (score: \pm 0 ). c Histological classification (score: +1 ). d Lymphatic invasion (score: +2 )

poorly differentiated adenocarcinoma, the risk of lymph node metastasis is believed to be high [2-5]. In agreement with other work, in this study, histological classification of submucosally invasive gastric cancer at the site of invasion (specifically, undifferentiated) was significantly correlated with lymph node metastasis on univariate analysis.

In studies in which advanced gastric cancer and submucosally invasive gastric cancer were examined by mucosal phenotype, cancer with gastric-type mucosal features has been sporadically reported to have a high prevalence of lymph node metastasis compared to cancer with intestinaltype mucosal features $[11,12]$. Even in the present study, the prevalence of lymph node metastasis of cancer with gastrictype mucosal features appeared to be higher than that of cancer with intestinal-type mucosal features, although no significant difference was found in prevalence of lymph node metastasis between the two factors.

Cancer with lymphocytic infiltration appears in various organs. In the digestive tract, gastric cancer has often been reported to exhibit lymphocytic infiltration. It is generally recognized that lymphocytic infiltration in cancer lesions inhibits cancer progression and lymph node metastasis in cases showing good prognosis and low prevalence of lymph node metastasis $[13,14]$. We therefore examined the 


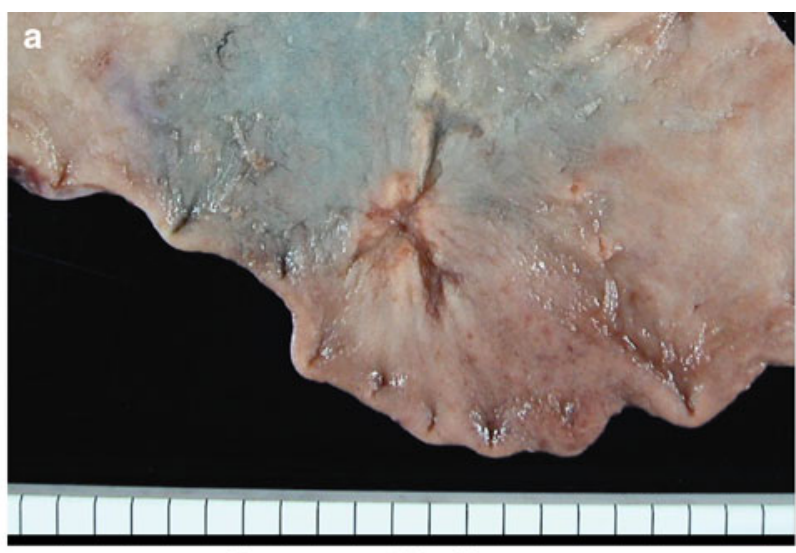

Tumor axes; $31 \times 16 \mathrm{~mm}$

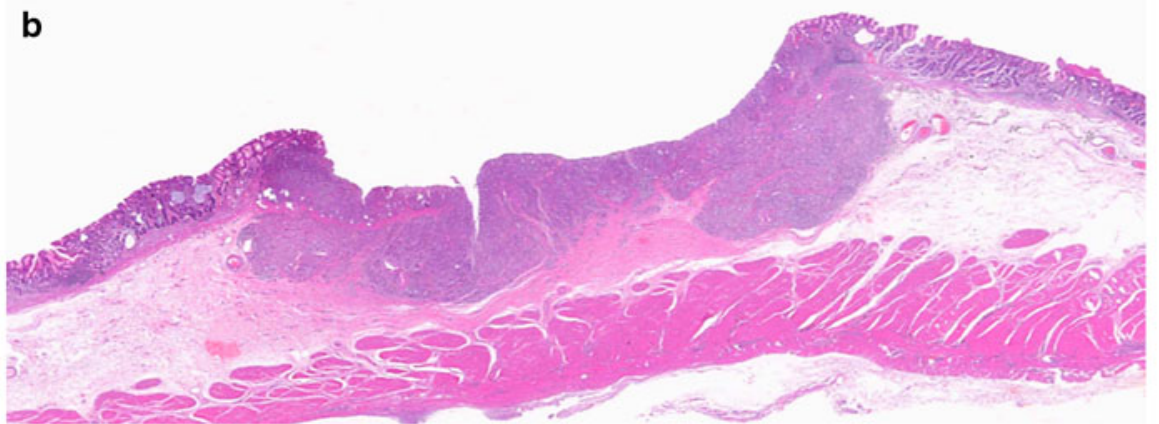

depth of submucosal cancer invasion $2750 \mu \mathrm{m} / \mathrm{UL}(+)$

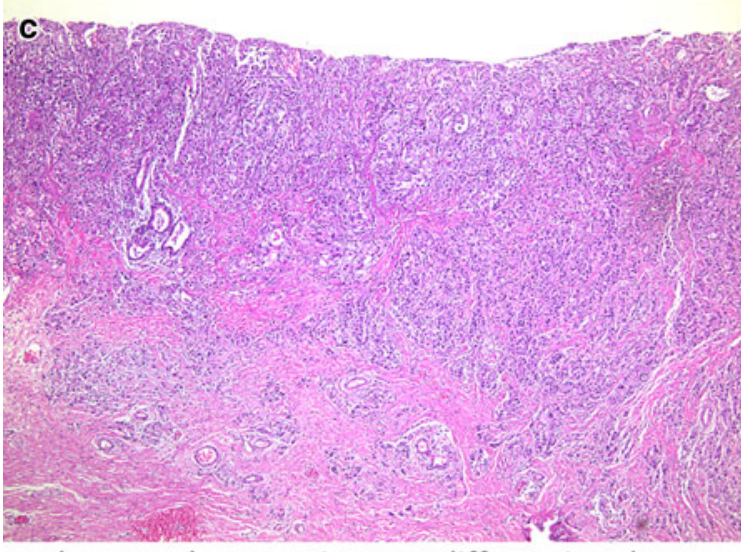

submucosal cancer tissue undifferentiated type

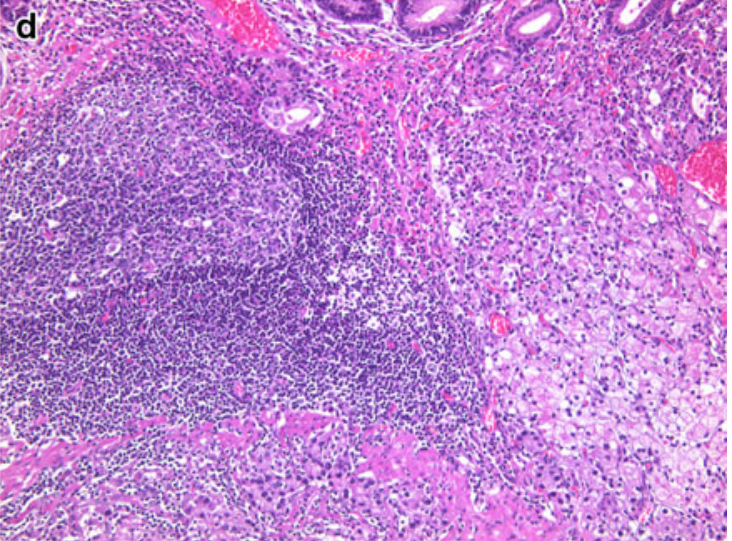

lymphocytic infiltration positive

Fig. 3 Case 2, which was negative for lymph node metastasis (total score: +1 ). a Tumor size (ulceration or its complication: score: +1 ). b Depth of submucosal cancer invasion (score: +1). c Histological classification (score: +1). d Lymphocytic infiltration (score: -2)

relationship between lymphocytic infiltration of cancer lesions and lymph node metastasis, finding that patients without lymphocytic infiltration in cancer lesions were at high risk for lymph node metastasis, with significant correlations found on both univariate and multivariate analyses.

Intralesional ulcers or ulcerous scars in early cancers are rare in cases of colonic cancer but frequent in gastric cancer. In the present study, intralesional ulcer was found to be a risk factor for lymph node metastasis on univariate analysis, but it was not an independent factor on multivariate analysis.

Because lymph node metastasis is induced by cancer that reaches the regional lymph nodes through the lymph ducts, lymphatic invasion is considered the most important risk factor for lymph node metastasis [1-6]. Even in the multivariate analysis in the present study, lymphatic 
invasion was found to be an important risk factor for lymph node metastasis. However, there were three lymphatic invasion-free patients who were positive for lymph node metastasis, thus deeming sensitivity of diagnosis of lymph node metastasis using lymphatic invasion as a risk factor to be $<100 \%$.

The rate of lymph node metastasis in venous invasionpositive patients was $30.4 \%$. Univariate analysis revealed a significant correlation of venous invasion with lymph node metastasis. The specificity of this parameter was high compared to that of lymphatic invasion, although venous invasion was not identified as an independent factor on multivariate analysis. Venous invasion can be diagnosed with relative ease using elastic fiber-staining methods with high reproducibility and objectivity. For these reasons, venous invasion appears to be important as a risk factor.

Univariate analysis of pathological factors in the present study revealed that tumor minor axis length, depth of submucosal invasion, histological classification of submucosally invasive gastric cancer (undifferentiated) at the site of invasion, lymphocytic infiltration, ulceration or scar in the lesion, lymphatic invasion, and venous invasion were significantly correlated with lymph node metastasis. In addition, multivariate analysis of these factors revealed that lymphatic invasion and lymphocytic infiltration were significant, independent risk factors for lymph node metastasis. On the basis of these findings, a pathological factorbased scoring system was devised to determine the need for additional surgical gastrectomy following endoscopic resection of submucosally invasive gastric cancer (Table 5). Scores for 130 patients included in the present study indicated that a total of 61 patients should have undergone additional gastrectomy after endoscopic resection of lesions (because they could be presumed to have lymph node metastasis), including all 22 patients with lymph node metastasis, and that only 39 patients were overtreated. The criteria based on the score showed a sensitivity of $100 \%$, specificity of $63.9 \%$, and rate of correct diagnosis of $70.0 \%$. Thus, the scoring system proposed in the present study is superior to the conventional standards because it retains high specificity, drastically decreases overtreatment of patients, and maintains sensitivity at $100 \%$ (Table 7).

The pathological factor-based scoring system proposed in the present study can be improved and made more precise by repeated application of the methods used here as more cases are accumulated.

\section{Case presentation}

Case 1, which was positive for lymph node metastasis (total score: +3 ). High risk of lymph node metastasis was indicated in this case because the total score was $>3$ (Fig. 2).

Case 2, which was negative for lymph node metastasis (total score: +1 ). Low risk of lymph node metastasis was indicated in this case because the total score was $<3$ (Fig. 3)

\section{Conclusion}

A scoring system was devised for additional gastrectomy following endoscopic resection on the basis of prediction of lymph node metastasis. This scoring system enables more precise selection of cases and may be useful in determining treatment for submucosally invasive gastric cancer following endoscopic resection.

Acknowledgments My deepest appreciation goes to Prof. Kazuhide Higuchi (Second Department of Internal medicine, Osaka Medical College, Japan) whose comments and suggestions were of inestimable value for my study. Special thanks also go to many doctors and staff members of the Department of Pathology, Osaka Medical College, Japan, whose valuable efforts made enormous contributions to my work.

\section{References}

1. Guidelines for Diagnosis and Treatment of Carcinoma of the Stomach. The Japanese Gastric Cancer Society; 2004.

2. Gotoda T, Sasako M, Yanagisawa A, et al. Incidence of lymph node metastasis from early gastric cancer; estimation with a large number of cases at two large centers. Gastric Cancer. 2000;3:219-25.

3. Seto Y, Shimoyama S, Kitayama J, et al. Lymph node metastasis and preoperative diagnosis of depth of invasion in early gastric cancer. Gastric Cancer. 2001;4:34-8.

4. Park DJ, Lee HK, Lee HJ, et al. Lymph node metastasis in early gastric cancer with submucosal invasion: feasibility of minimally invasive surgery. World J Gastroenterol. 2004;10:3549-52.

5. Abe N, Watanabe T, Suzuki K, et al. Risk factors predictive of lymph node metastasis in depressed early gastric cancer. Am J Surg. 2002;183:169-72.

6. Japanese Classification of Gastric Carcinoma, 14th edn. Japanese Gastric Cancer Association; 2010.

7. Japanese Classification of Colorectal Carcinoma, 8th edn. Japanese Society for Cancer of the Colon and Rectum; 2009.

8. Statview [computer program]. Version 5.0 Berkeley, CA, USA: Abacus Concepts; 1998.

9. Lins RL, Elsevieers M, Daelemans R, et al. Prognostic value of a new scoring system for hospital mortality in acute renal failure. Clin Nephrol. 2000;53(1):10-7.

10. Adnan I, Gene YS, Alexander YR, et al. Early identification of patient at risk for symptomatic vasospasm after aneurysmal subarachnoid hemorrhage. Crit Care Med. 2000;28(4):984-90.

11. Koseki K, Tanizawa T, Koike M, et al. Distinction of differentiated type early gastric carcinoma with gastric type mucin expression. Cancer (Phila). 2001;89:724-32.

12. Saito A, Shimoda T, Nakanishi Y, et al. Histologic heterogeneity and mucin phenotype expression in early gastric cancer. Pathol Int. 2001;51:165-71. 
13. Lertprasetsuke N, Tsutsumi Y. Gastric carcinoma with lymphoid stroma. Analysis using mucin histochemistry and immunohistochemistry. Virchows Arch A Pathol Anat Histopathol. 1989;414(3): 231-41.
14. Watanabe H, Enjyoji M, Imai T. Gastric carcinoma with lymphoid stroma. Its morphologic characteristics and prognostic correlations. Cancer (Phila). 1976;38(1):232-43. 